\title{
Colaboración y redes socioeducativas para la inclusión de la infancia vulnerable
}

\section{Collaboration and social-educational networks for the inclusion of vulnerable children}

\author{
Begoña Martínez Domínguez ${ }^{1}$ \\ begona.martinez@ehu.eus \\ Israel Alonso Sáez \\ israel.alonso@ehu.eus \\ Isabel Martínez Domínguez \\ isabel.martinez@ehu.eus \\ María José Alonso Olea \\ josebe.alonso@ehu.eus \\ Universidad del País Vasco, España
}

\section{Resumen:}

Existe un reconocimiento social y profesional sobre la necesidad de que todos los agentes y entidades sociales y educativas que intervienen con la infancia vulnerable en riesgo trabajen en colaboración para mejorar su protección e inclusión. La colaboración entre los ámbitos social y educativo, puede articularse a través de un trabajo en red-formal e informal- y a nivel macro, meso y micro, de formas muy diferentes en función de cada territorio, aunque de cada experiencia es posible inferir pautas para avanzar en otros.

El artículo forma parte de una investigación en progreso realizada en el País Vasco dentro del marco de un proyecto coordinado más amplio sobre redes de innovación para la inclusión social y educativa. Analiza el proceso de construcción y desarrollo de una red socioeducativa para la infancia vulnerable surgida en un

\begin{abstract}
:
There is a social and professional understanding that the stakeholders and the social and educational entities that work with vulnerable and at-risk children need to collaborate to better ensure that such children are protected and included. CoIlaboration between social and educational agents can be achieved through both formal and informal networks that work at the macro, meso and the micro levels and in different ways depending on the autonomous community they operate in, and each experience can provide lessons for moving forward in other settings.

This article is part of an on-going research project being carried out in the Basque Country, within a broader and coordinated project on social and educational innovation networks for inclusion. It analyses the process of building and developing a social-educational network for vulnera-
\end{abstract}

1 Dirección para correspondencia (correspondence address):

Begoña Martínez Domínguez. Dpto. de Didáctica y Organización Escolar. Universidad del País Vasco. Facultad de Educación, Filosofía y Antropología. Edif. 1. Avd. de Tolosa, 70. 20018 San Sebastián (España). 
contexto singular. Los resultados ponen de manifiesto la relevancia de esta forma alternativa de acción socioeducativa, así como las dificultades y retos que plantea. Aunque en la literatura científica no queda bien identificada la relación mutua entre las redes formales e informales, esta contribución ofrece argumentos fundamentados para la integración de ambas. Por consiguiente, es necesario integrar la dimensión profesional, comunitaria y política en cualquier proyecto que busque la cohesión social.

\section{Palabras clave:}

Colaboración; Redes socioeducativas; Inclusión socioeducativa; Infancia vulnerable. ble children within a specific context. The results point to the value of this alternative form of social-educational action, as well as the difficulties and challenges it poses. Although the interrelationship between formal and informal networks is not clearly established in the literature, our contribution offers solid arguments for integrating them. Any undertaking that seeks social cohesion must integrate professional, community and policy dimensions.

\section{Key words:}

Collaboration; Socio-educational network; Socio-educational Inclusion; Vulnerable childhood.

\section{Résumé:}

Il y a un consensus social et professionnel sur la nécessité pour tous les acteurs et organismes sociaux et éducatifs travaillent ensemble, et interviennent auprès des enfants vulnérables en situation de risque afin d'améliorer leur protection et leur inclusion. La collaboration entre tous les intervenants sociaux et éducatifs peut se structurer à travers le travail en réseau - formel et informel - et, aux niveaux macro-méso-micro, de multiples façons différentes en fonction de chaque territoire, bien qu'il soit possible de déduire de chaque expérience des modèles qui contribueront à progresser dans d'autres domaines. L'article fait partie d'une recherche en cours au Pays basque (espagnol) qui s'inscrit dans le cadre d'un projet plus ample et coordonné sur les réseaux d'innovation aux fins de l'inclusion sociale et éducative. L'étude analyse le processus de construction et de développement d'un réseau socio-éducatif pour l'enfance vulnérable dans un contexte spécifique. Les résultats mettent en évidence l'importance de cette formule alternative d'action socio-éducative, avec les difficultés et les enjeux qu'elle comporte. Bien que dans la littérature scientifique la relation mutuelle entre les réseaux formels et informels ne soit pas clairement identifiée, notre contribution offre des arguments fondamentaux pour leur intégration. Par conséquence, il est nécessaire d'intégrer la dimension professionnelle, communautaire et politique dans tout projet qui vise la cohésion sociale.

\section{Mots clés:}

Collaboration; Réseaux socio-éducatifs; Inclusion socio-éducative; Enfance vulnérable.

Fecha de recepción: 7-2-2018

Fecha de aceptación: 23-4-2018

\section{Planteamiento del problema}

\section{La Convención sobre los Derechos del Niño (en adelante, CDN) del año 1989 reconoce a la persona menor de edad, como sujeto de derechos, así como la necesidad de ser protegida debido a su situación de vulne-}


rabilidad. Sin embargo, la situación de la infancia en el mundo, no está libre de amenazas y peligros de desamparo, abandono y dificultades. La compleja sociedad actual, donde la desigualdad es una realidad social en aumento, la precariedad de la vida, la violencia que genera y la falta de recursos de las familias y comunidades hacen que los derechos de la infancia no estén siendo asegurados ni protegidos. Por ello, en cualquier sociedad justa y democrática es un imperativo que el trabajo socioeducativo garantice su desarrollo digno y sano, para que pueda lograr una ciudadanía plena sin riesgos de exclusión.

Este artículo corresponde a una investigación en proceso que se está desarrollando en el País Vasco sobre Redes de innovación para la inclusión socioeducativa de la infancia vulnerable ${ }^{2}$, dentro de un proyecto coordinado entre cuatro universidades (Vigo, Cantabria, Sevilla y País Vasco) sobre procesos y proyectos de innovación acerca de la creación de contextos participativos referidos a comprender, situar y potenciar transformaciones socio-educativas inclusivas y sostenibles.

El proyecto del País Vasco versa sobre la mejora de la infancia y familias vulnerables. Se ha tomado en consideración, de un lado, resultados de dos investigaciones previas (Haurbabesa Lanbide, 2011; Martínez, B. et al., 2015). Se concluyó que el avance en la inclusión de la infancia y juventud, en la que intervienen diversos profesionales y entidades socioeducativas en nuestro territorio, exige reforzar la colaboración entre los agentes y el protagonismo de las voces de los sujetos con una perspectiva innovadora y comunitaria. De otro, un par de estudios recientes (SIIS, 2017; UNICEF, 2017) que han documentado un panorama ambivalente en el País Vasco. Ciertos indicadores del bienestar de la infancia son mejores que los de otras Comunidades Autónomas pero, a raíz de la crisis económica, revelan como en otros contextos un estancamiento y riesgo de retroceso en materia de inclusión y protección social.

En nuestra comunidad existen varias leyes en la materia ${ }^{3}$ pero no algún organismo con competencias integrales. La salud, educación, vi-

2 Redes de Innovacion para la Inclusion Educativa y Social: Infancia Vulnerable, Servicios Socioeducativos y Familias (EDU2015-68617-C4-2-R) (MINECO/FEDER, UE).

3 Ley 3/2005, de 18 de febrero, de Atención y Protección a la Infancia y la Adolescencia, de Euskadi. Ley 12/2008, de 5 de diciembre, de Servicios Sociales. Ley 13/2008, de 12 de diciembre, de Apoyo a las Familias. Ley Orgánica 8/2015, de 22 de julio, de modificación del sistema de protección a la infancia y a la adolescencia. Decreto 185/2015, de 6 de octubre, de cartera de prestaciones y servicios del Sistema Vasco de Servicios Sociales. Ley 2/2016, de 7 de abril, de Instituciones Locales de Euskadi. 
vienda y diversos servicios sociales son objeto de políticas sectoriales dirigidas particularmente a la infancia, resultando todavía un reto el diseño y la realización de actuaciones transversales, capaces de responder globalmente a las realidades y necesidades de sujetos, familias y colectivos más vulnerables. Sobre ese contexto de fondo, nuestra investigación se acoge a una perspectiva más integral y pretende ofrecer cómo puede llevarse a cabo en un ámbito municipal concreto. Se trata de reconocer y valorar actuaciones coordinas valiosas que vienen desarrollándose dentro del mismo y explorar, a su vez, las posibles contribuciones que pueda tener una mayor coordinación de los diferentes servicios y agentes involucrados.

\section{Antecedentes y fundamentación teórica}

Infancia y vulnerabilidad son dos términos que, a pesar de sus significados difusos, se emplean en la literatura científica actual para hacer referencia al concepto de persona menor de edad, desde el nacimiento hasta los dieciocho años, tal como se recoge en la citada Convención. Se trata, por tanto, de una etapa clave de la vida, del desarrollo, socialización y educación de los individuos, en la que los menores dependen de las personas adultas. Lo que convierte a la infancia en una etapa de vulnerabilidad potencial, cuyo grado mayor o menor dependerá de múltiples factores familiares, sociales, culturales, económicos y otros (CDN, 1989; Jolonch, 2002; Kim, 2014), en la que los adultos involucrados deben ser sus protectores.

Cuando, en una sociedad, los mecanismos de protección de la persona menor no garantizan sus derechos esenciales, se pueden provocar exclusiones intolerables. Por contra, cualquier sociedad que aspire a ser inclusiva y justa, debe articular los sistemas de protección necesarios para garantizar los derechos de la infancia. Y comprender las situaciones de vulnerabilidad desde una perspectiva social y comunitaria constructiva, en lugar de problematizar al sujeto como responsable de una situación en la que, en gran medida, es la víctima. Por tanto, el bienestar de la infancia es una cuestión social (Castel, 1997) y por ello debería ser una prioridad sociopolítica en todo el mundo (Prilleltensky, 2010).

Aunque deben reconocerse los esfuerzos realizados y los logros alcanzados, sigue siendo un reto de la sociedad actual la lucha por la 
inclusión y el bienestar de la ciudadanía en la infancia. A pesar de los avances notables en materia de educación inclusiva, como se aprecia en la literatura especializada (Booth y Ainscow, 2015, Escudero y Martínez, 2011; Parrilla, Muñoz y Sierra, 2013; Sapon-Shevin, 2013), aquella es un aspecto de la inclusión en la sociedad. Ello exige la adopción de un enfoque cuyos principios lleven a acometer simultáneamente actuaciones de mejoras educativas y sociales. Siendo una tarea ciertamente compleja, tanto teórica como prácticamente, son fundados los acuerdos de entender la inclusión como un proceso que exige la participación de todas las personas, escuchando la voz de los diversos agentes implicado, y con los propósitos de crear sistemas participativos regidos por los valores propios de una sociedad inclusiva (equidad, aceptación de la diversidad, igualdad de oportunidades, justicia social...). Ello implica avanzar en una nueva cultura basada en la participación de la comunidad y echar mano de ideas y herramientas de cambio focalizadas en lo local, de forma que la democratización de los procesos socioeducativos constituya elementos nucleares para la cohesión social. De ese modo, la articulación de redes educativas y sociales, efectivamente participativas y propiciando relaciones estrechas entre escuela y comunidad adquieren un papel relevante (Muñoz, Raposo, Doval y Parrilla, 2011). Pueden posibilitar que la educación inclusiva, entendida como una cuestión de derecho, conecte con la democracia, la justicia y la equidad. Bajo esa perspectiva, los avances por alcanzar lo que respecta a la inclusión de la infancia pueden favorecerse mediante un trabajo colaborativo entre diferentes actores, entidades e instituciones. La tesis principal de este artículo es que la existencia de redes -formales e informales - que combinen, dentro de un determinado territorio, lo global y lo local, los planos normativos y las prácticas macro, meso y micro, pueden contribuir a proteger socialmente a la infancia en situación de vulnerabilidad, apostando por evitar o, cuando menos, disminuir los riesgos de exclusión social que la afectan.

En los últimos años se viene proponiendo una concepción del trabajo en red como:

...un modelo de organización alternativo a los tradicionales organigramas jerárquicos, con capacidad para integrar instituciones y actores con responsabilidad educativa en un plano de corresponsabilidad, unidos por el interés común, primero para compartir el análisis de las necesidades y los proyectos 
de un territorio o comunidad y, segundo, para coordinar la acción socioeducativa de un modo integral y coherente (Longás, Civís y Riera, 2008, p.306).

En ese sentido, la noción de red socioeducativa lleva a reconocer que los servicios y recursos disponibles suelen ser provistos por diferentes redes como la educativa, la de los servicios sociales y la sanitaria. Pareciendo obvio que no es posible intervenir profesionalmente fuera de alguna red ni, tampoco, al margen de las restantes (Ubieto, 2009). Sin embargo, existe cierta confusión respecto al uso y significado que, tanto en el mundo anglosajón como francófono, se atribuye a términos aparentemente sinónimos como coordinación, colaboración, integración de servicios u otros similares. De hecho, la perspectiva y los resultados de los que se ocupa este artículo responden a una determinada visión del trabajo de los diferentes profesionales que trabajan con la infancia. Susceptible de situarse a lo largo de un continuo sucesivo y crecientemente integrador, desde aquellos modelos que suponen: una forma de intervención aislada, alguna coordinación, colaboración, y alguna modalidad de red socioeducativa. Son las dos últimas las que propiamente nos interesan aquí y comportan, cada una a su manera, el propósito de construir una mirada colectiva y comunitaria de la infancia y el trabajo con ella. Avanzar por ese camino requiere un determino tipo de ideas, y procesos a través de los cuales se promuevan innovaciones que exploren nuevas formas de intervención en ese ámbito.

Entendidas las innovaciones como procesos, no como actuaciones instantáneas, suele identificarse una primera etapa, consistente en la construcción del caso en red (Gutiérrez y Sánchez, 2017; Ubieto, 2009). Ello implica que diferentes profesionales ponen a la persona que atienden desde distintos sistemas en el centro de atención del trabajo. Como apunta Ubieto (2009), esa etapa se inicia con alguna pregunta, reveladora del reconocimiento de un cierto vacío, un "no saber", asumiendo, que las respuestas por encontrar han de ser construidas desde y con un conocimiento colectivo. A través de la horizontalidad, suspendiendo las definiciones previas y los prejuicios sobre las personas con las que se pretende trabajar y reconociendo sus capacidades. Según el autor, cualquier proyecto en red de esta naturaleza debe incorporar cuatro ámbitos: la asistencia, la formación, la investigación y lo comunitario.

En una etapa más avanzada, se trataría de asumir la necesidad de interacción y diálogo, dos procesos clave de cara a articular, con propó- 
sitos de mejora y transformación, los dispositivos y las acciones a llevar a cabo con la infancia en riesgo y sus familias (Castillo, 2009). De manera que la acción socioeducativa no solo exige la coordinación entre diferentes profesionales, sino más bien la creación de una comunidad de aprendizaje entre diferentes agentes, profesionales y participantes, con una visión comunitaria e innovadora del trabajo socioeducativo, más allá del aislamiento y la mera coordinación (Castillo, 2009; Ballester, 2014).

Recurriendo al marco de referencia planteado por las denominadas "comunidades de práctica" (Wenger, 2001), e incluyendo aquellas concepciones que definen el desarrollo y aprendizaje humano como procesos socioculturales y situados de construcción del conocimiento y el uso del mismo (Brown, Collins y Duguid, 1989), se trata de que los profesionales creen y compartan significados comunes, modos de hacer y herramientas compartidas, apoyos mutuos y compromisos efectivos con el diseño y la implementación de proyectos socioeducativos. Díaz y Cívis (2011) insisten en lo mismo al caracterizar el trabajo en red como un cambio que permite incorporar la mirada de todos los agentes socioeducativos de la comunidad para afrontar el reto de la educación de forma conjunta y eficiente, generando sinergias y evitando la infravaloración y el solapamiento de los servicios. Se trata de redes socioeducativas organizadas y vertebradas en torno a la colaboración de sus miembros, la definición conjunta de metas u objetivos y la adopción compartida de actuaciones, basadas en la creación y aplicación comunitaria de conocimientos e innovaciones. En tales redes, el flujo de información y el capital social tienen un papel central, así como las estructuras dentro de las que operan y las acciones de los sujetos que las habitan. Y, desde luego, los principios éticos de actuación y los elementos cognitivos y emocionales de los agentes involucrados. En ese sentido, son relevantes los principios de proximidad, transversalidad, participación, cooperación y proyección (Díaz, Civís, Longás y López, 2010, Civís y Longás, 2015).

En la tercera fase de la innovación, correspondiente a la implementación, serán consecuentes, entonces, aquellas estrategias que propicien una acción integral y conjunta, corresponsable, eficiente y sostenible en un territorio determinado (Cusso, Longas y Riera, 2015; Milward y Provan, 2006). Porque: 
"Si los retos son complejos, las respuestas no pueden ser simples o fragmentadas y, en este sentido, las estructuras en red permiten organizarlas desde una visión holística y sistémica de la realidad" (Civis y Longás, 2015, p. 232)

\section{Diseño y metodología}

Dentro del proyecto general diseñado para el período 2016-2019, la fase que aquí nos ocupa corresponde a lo que se ha llevado a cabo en los dos primeros. Sus objetivos fueron "entrar en contacto, analizar y extraer conclusiones pertinentes sobre la génesis y desarrollo de un trabajo socioeducativo". Implica, por lo tanto, partir y reconocer actuaciones llevadas a cabo con la infancia vulnerable en un ámbito local (municipio) y construir con y sobre las mismas. De ahí que, en términos metodológicos, se haya optado por un enfoque cualitativo y participativo (Parrilla y Sierra, 2015) y el estudio de caso. Por la confidencialidad comprometida con los participantes $y$, asimismo, porque nuestro objeto de estudio no es tanto la infancia y las familias vulnerables como las dinámicas de colaboración a construir, baste señalar que nuestro contexto de investigación corresponde a un municipio de 11.000 habitantes del País Vasco.

La selección del mismo ha obedecido a dos razones. La primera se debe al hecho de que, al de estar separado por una carretera nacional, se han generado dos poblaciones diferentes en socialización y un desarroIlo desigual: la "baja", más acomodada y la "alta", en situación de mayor vulnerabilidad. La segunda, derivada de la anterior, la preexistencia, desde hacer varias décadas, de una trayectoria de trabajo comunitario que ha permitido reducir progresivamente muchas desigualdades. En ese contexto y gracias al impulso de un grupo político independiente que ha ido ganando sucesivas elecciones, se creó una Comisión Socioeducativa constituida por diferentes entidades y agentes implicados en afrontar las necesidades de la infancia vulnerable y sus familias.

El trabajo de campo realizado surge con la entrada y negociación en el municipio y culmina con la integración del equipo investigador en dicha Comisión. Ello ha permitido una recogida de la información aplicando distintas técnicas y análisis como se muestra a continuación: 


\section{Análisis de contenido de documentos primarios y secundarios \\ -Actas de las reuniones de la Comisión Socioeducativa (2006-2016) \\ -Planes de Convivencia (2012-2017) \\ -Documentos generados por la Comisión y páginas web de las entidades integrantes}

\section{Entrevistas semi-estructuradas \\ (20 informantes clave de la comunidad que trabajan con la infancia, cuyos perfiles y códigos (entre paréntesis) aparecen en el cuadro siguiente:}

\section{Dinámicas participativas}

realizadas en sesiones de trabajo con la Comisión
*ÁMBITO POLÍTICO (AC): Alcalde y Concejales de educación y de cultura.

* ÁMBITo EDUCATIVo (AE): Directora de CEIP, Directora y Orientador de IES, Profesorado en activo y jubilado.

*VOLUNTARIADO (V): Madre de la AMPA, Dinamizador comunidad cristiana, Educadoras de comedor y apoyo escolar, Coordinadora de catequesis.

*ÁMBITO SOCLAL (AS): Educadores de EISE y Trabajadora social.

* OTROS SERVICIOS (OS): Pediatra y Policia municipal.

Figura 1. Técnicas de recogida de información.

La aplicación de las técnicas señaladas ha permitido recabar información variada sobre problemas de la infancia-adolescencia y sus familias y el trabajo en red de los servicios socioeducativos. Se realizaron sesiones de trabajo y entrevistas semi estructuradas, con dinámicas abiertas para que los participantes aportaran libremente sus puntos de vista. Las informaciones aportadas los participantes fueron grabadas de común acuerdo, transcritas y trianguladas con la procedente de las actas aportadas por la Comisión Socioeducativa (red formal existente). Para el análisis del contenido se construyeron inductivamente categorías y códigos (Andréu, 2000) procesados a través del programa NVIVO10. El sistema categorial construido ha permitido elaborar resultados relacionados con etapas, hitos, dificultades y oportunidades.

\section{Resultados}

Aunque es difícil establecer fechas precisas de sus inicios y términos, se ha podido observar la existencia de dos etapas de colaboración, enca- 
denadas en el tiempo, una formal y otro informal. Considerando las limitaciones de espacio disponible, se presentan los resultados obtenidos en las dimensiones citadas articulados en las dos etapas y siguiendo un orden cronológico. Dada la singularidad del municipio y la confidencialidad comprometida con los participantes, en la presentación de los resultados no aparecen datos específicos referidos a la infancia vulnerable ni citas singulares a informantes que llevarían fácilmente a identificarlos.

\section{$1^{\text {a }}$ ETAPA: Desarrollo de una cultura de colaboración comunitaria a través de redes informales}

Esta etapa se extiende, aproximadamente, entre los años 80 y 90. Se caracteriza por la participación de una buena parte de la ciudadanía que, en gran medida, surgió de forma "natural" ante la gravedad y proximidad de las necesidades entonces planteadas, a las que se hizo frente, mediante redes informales construidas con agentes educativos tradicionales y los incipientes servicios sociales, expresamente creados para atender a la infancia vulnerable y sus familias.

En la década de los años 80, con la crisis industrial y las necesidades económicas provocadas por aquella, surge en este municipio la semilla que ayuda a construir el trabajo en red desde una perspectiva comunitaria. Impulsados por la Iglesia, los maestros, jóvenes y personas voluntarias de la parte alta del municipio, realizan diferentes actuaciones solidarias, con la colaboración de líderes de la comunidad que comparten un mismo objetivo. A saber, contribuir en la mejora de problemas graves de subsistencia que tiene una parte muy importante de la población. Como respuesta al estado de abandono urbanístico y social de esta parte de la población, un grupo de dichos líderes naturales se constituye en un partido local independiente que en el año 1991 gana las elecciones municipales.

Como se aprecia en el testimonio aportado por uno de uno de los informantes del ámbito político, una de las primeras actuaciones de esta agrupación fue hacer frente y apoyar la situación de las familias que tenían una fuerte desestructuración y marginalidad.

"Bueno, en principio nosotros cuando entramos como grupo político vecinal lógicamente éramos un grupo de ciudadanos, de vecinos y nos preocupaba la gente. Entonces había una serie de familias desestructuradas...y nos dimos cuenta de que 
había que actuar con ellas...porque ello suponía una especie de inversión para el ayuntamiento ya que en la medida en que las recuperaras podrías evitar otros problemas para el resto" (AP)

En este sentido, es clave la apuesta que se hace en 1992 por dotar al municipio de un Equipo de Intervención Comunitaria ${ }^{4}$ (EIC), al tratarse de una situación de emergencia social muy grave:

"Había niños por la calle ..., había llegado una serie de gente de aluvión y con unas actividades poco honestas, con tráfico de droga y dinero fácil... veíais el contrasentido de niños con un billete de 1.000 pesetas que iban a comprar chucherías en calzoncillos...para evitar hacer en casa la comida... Se acercó la policía y me dijo: "vemos niños que van al centro solo a comer... salen del comedor a las 3 y se vuelven a la calle sin que nadie se preocupe por ellos" (AE)

En este contexto, desde el ayuntamiento se considera clave contar con la colaboración de la policía municipal para controlar la asistencia regular de la infancia vulnerable a la escuela. $Y$ al mismo tiempo dotar al municipio de mayores servicios y recursos para el ocio y tiempo libre y de instalaciones deportivas que no existían. Como veremos en la siguiente voz, se tenía claro que todas las medidas se tenían que plantear con una intención educativa y teniendo en cuenta a toda la ciudadanía,

"ya no es solamente que intentemos mejorar con un equipo socioeducativo, sino que la parcela del bienestar social crezca; ...en cultura se necesitan técnicos que promocionen actividades. y que éstas sean el contrapunto a otras actividades no tan recomendables" (AP)

En ese mismo sentido, cabe destacar cómo el ayuntamiento reconoce y pone en valor el trabajo que venían realizando profesionales y personas que, de forma voluntaria, prestaban apoyo a la comunidad, incorporándolas a los nuevos proyectos institucionales. Por ejemplo, se reconoció una Fundación surgida desde la parroquia para trabajar en la parte

4 A finales de los 80 y principio de los 90, comenzaron a crearse en algunos municipios de Bizkaia y algunos barrios de Bilbo, los Equipos de Intervención Comunitaria (EIC) pertenecientes a diversas entidades, formados principalmente por tres figuras: educador/a de familia, educador/a de calle, animador/a comunitario. 
alta con la infancia y juventud vulnerable en el Tiempo Libre educativo, firmando un convenio para ofertar programas y servicios dirigidos a toda la comunidad.

"hay un momento importante en el que se encuentran otras sinergias con otras entidades sociales...era una oportunidad que la Fundación ofreciera dos programas para la atención a preadolescentes y adolescentes y jóvenes desde el ámbito no formal, aunque nos costó bastante a nivel administrativo" ( $)$

Enlazando con la voz anterior, hay tres aspectos de los procesos de regularización de las actuaciones socioeducativas cuando surgen las redes formales que quisiéramos destacar, pues son numerosos los testimonios recogidos que los avalan.

El primero, el riesgo de que se fragmenten las intervenciones diferenciando ámbitos, programas y profesionales que, a la postre, burocraticen procedimientos que impiden intervenciones más integrales y comunitarias que suelen ocurrir cuando se cuenta con el apoyo de redes de carácter informal. Sirva como muestra la experiencia vivida por educadores sociales que, como miembros del primer equipo, actuaban ante las necesidades detectadas en la comunidad, aportando la inmediatez y la adaptación de la respuesta con una mirada más holística que la que más tarde pueden ofrecer.

"Es que al principio... a los programas de las ludotecas o las colonias de día iban los educadores, se acercaban allí y entonces era un momento para que también la parte del colegio fuera a un ámbito más informal y poder contactar con estos críos y crías" (AE)

El segundo aspecto hace referencia a la necesidad de revisar e integrar los modelos de intervención que, de forma explícita y/o implícita, llevan a cabo los agentes y servicios socioeducativos cuando pasan de mantener una colaboración más informal a formar parte de una red estructurada; dado que puede reforzar o cambiar la orientación de los mismos, sobre todo cuando esta última surge de "arriba a abajo". O, también, cuando se modifican los marcos normativos que los regulan. En este sentido, como veremos después, la aparición de los nuevos Equipos de Intervención Socioeducativa (EISE) es una muestra de ello, cuando 
desaparece la figura de la Animación Comunitaria y se pierde el enfoque comunitario que tenía el EIC en la etapa anterior.

El tercer y último aspecto que quisiéramos señalar es que una red formal de colaboración auténtica supone por principio, modos de trabajar horizontales, dialogados, basados en la confianza y el establecimiento de vínculos afectivos, aspectos que son básicos en las relaciones propias de redes informales. En este sentido, vemos como la creación de redes socioeducativas eficaces, además de contar con el apoyo necesario de las correspondientes administraciones, necesitan prestar una atención especial a que la colaboración de los profesionales y servicios del ámbito educativo formal y no formal sean, en efecto, horizontales. Ello es preciso de cara a soslayar diferencias de status y culturales profesionales, que pueden dificultar la colaboración auténtica y, como consecuencia, la buena atención debida, acorde con los derechos que tienen la infancia vulnerable y sus familias. Como dice un informante:

“Deberíamos aprender, todos los ayuntamientos, que tú tienes que ser una parte más que colabore en ese proceso, y una vez que entendamos eso, todos seremos capaces de llevar al pueblo hacia delante y a la juventud también" (AP)

En síntesis, podemos decir que todas las acciones mencionadas, aunque no hayan estado articuladas en un plan comunitario de actuación, fueron sin duda, el germen necesario y propicio para el inicio de un modelo de participación "de abajo a arriba". Ya que, como se ha observado en otros casos (Díez et al., 2010), en este municipio el sentimiento de pertenencia e identidad dio lugar a una red informal poco común, construida sobre colaboraciones múltiples entre políticos, instituciones y ciudadanía. Esta, ha contribuido a conseguir un determinado desarrollo comunitario y, como veremos en la segunda etapa, la formalización de una red que permite seguir avanzando en la mejora de la atención de la infancia y las familias más vulnerables.

\section{ETAPA 2: Trabajo colaborativo en red(es) formal(es).}

La segunda etapa mencionada, que abarca los últimos años del siglo pasado y lo que va del actual, se caracteriza por la colaboración de antiguos y nuevos agentes del ámbito educativo y social, que intervienen 
con la infancia vulnerable y sus familias. En sus inicios es relevante la creación de una Comisión de Convivencia, que ha ido conformándose como una red dentro del municipio, representando un avance destacable hacia lo que entendemos aquí como una red socioeducativa.

El primer hito relevante de esta segunda etapa viene dado por los inicios de un proceso inicial de transformación de la Escuela Pública del barrio alto en una Comunidad de Aprendizaje en 1998-99. Esta decisión, impulsada por el equipo directivo con el lema "se necesita un pueblo entero para educar a un niño o niña", favorece que los profesionales de la red escolar se acerquen formalmente a las familias más desvinculadas de la escuela, con el propósito explícito de implicarlas en la construcción conjunta del Proyecto Educativo. Se inicia así un proceso de participación que facilita el flujo de la información y corresponsabilidades entre la escuela y la comunidad.

Entre los años 2004-2006 surgen algunos conflictos graves con una parte de la infancia y jóvenes de la zona alta del municipio y se asume la necesidad de trabajar con una perspectiva educativa y comunitaria destinada a resolverlos:

"Había un grupito de jóvenes que habían tenido varios problemas... y nos vimos implicados bastantes agentes que trabajábamos con la educación. Así que propusimos ir al Ayuntamiento, juntarnos y ver cómo avanzar para acabar con todo eso" (AE)

El análisis colectivo de la situación y el impulso conjunto de la escuela, la parroquia y el EISE ${ }^{5}$ derivan, en 2007, en la creación de una Comisión Socioeducativa, dentro de cual varias entidades empiezan a trabajar de forma coordinada, poniendo en el centro de atención a las personas atendidas desde diferentes sistemas, servicios y profesionales. Es una etapa en la que el discurso de la inclusión sirve como referente y estímulo a respuestas coordinadas por parte de distintos

5 EISE surge a partir del DECRETO FORAL número 124/96, para desarrollar los programas del Plan de Intervención Socio-Educativa con Infancia, Juventud y Familia en el año 1997. Este equipo de intervención socioeducativa lo componen dos Educadores Sociales, uno de familia y otro de calle. Es un recurso municipal constituido como dispositivo especial y de refuerzo de los Servicios Sociales de Base, cuyo objeto es trabajar con menores de entre 0 y 18 años, y sus familias, considerados de riesgo de desprotección leve y/o moderada; a través de una intervención educativa en el propio contexto de vida y relación, centrada en el/la menor, su familia o ambos. 
profesionales especializados que trabajan con las personas en riesgo de exclusión.

Otro hito del proceso en el año 2008, es el inicio del trabajo en el municipio de un Centro especializado en resolución de conflictos. Impulsado por el Ayuntamiento y con la ayuda del Gobierno Vasco, se contratan los servicios de este centro de mediación en la resolución pacífica de los conflictos, como asesoramiento y asistencia técnica para del Plan de Convivencia. Esta decisión llevó a un diagnóstico de situación en el municipio, y a reforzar la composición de la Comisión con representantes institucionales de: Ayuntamiento, Servicios Sociales de Base, Equipos directivos y Amas de los tres centros escolares y entidades sociales sin ánimo de lucro para llevar a cabo programas socioeducativos de tiempo libre. Ello significó la oportunidad para dar un paso adelante con una mirada comunitaria.

Durante el curso 2011-12 tiene lugar, promovida por la política municipal, una primera decisión en pro de la fusión y el emplazamiento de los dos centros de primaria de la zona alta y baja. Se trataba de abogar por una mejor inclusión de la infancia construyendo una identidad cultural compartida. Aunque ello podía comportar el riesgo de que, por aumentar el tamaño y la diversidad de los agentes afectados, fuera más difícil gobernar el proyecto y la implicación de todos en el mismo, se asumió el reto en aras de la opción inclusiva tomada. La segunda, a su vez, consistió en la creación de un IES en la zona alta. Así, la infancia de las dos partes del municipio, desde los 6 hasta los 16 o 18 años, se relacionaba y aprendían juntos combatiendo la estigmatización de la zona más desfavorecida y la consiguiente guetización. Con similar filosofía, se tomaron decisiones sobre la localización de los recursos municipales para el tiempo libre:

"con los nuevos colegios, tanto el centro de primaria como el IES han provocado que todos se conozcan más. ¿Por qué? Porque independientemente de que las zonas del municipio estén al lado, te sientes al lado. Y resulta que, si vas a los grupos de tiempo libre y a un campamento y te encuentras con el alcalde como un monitor más repartiendo comida, pues pasa lo mismo". (AP)

El trabajo de la Comisión confirma la necesidad de dedicar tiempo al diálogo, la negociación de objetivos, el ajuste de los diferentes modelos de intervención, la coordinación de estructuras y acciones, poniendo una atención especial en criterios éticos y emocionales. Lo que 
no obvió, como puede suponerse, el surgimiento de comprensibles dificultades al lado de otras oportunidades. Entre ellas, las ligadas al momento crítico, ocurrido en 2013, cuando los componentes del EISE, promotores y miembros de la Comisión desde su creación, cuestionan su presencia en ella y su razón de ser. Uno de los motivos aducidos fue que, una vez desaparecidos los graves conflictos de la infancia que la provocaron, empezaba a no tener sentido. Así se expresa un informante:

"la comisión se Ilamaba de infancia porque tenía que ver con aquella situación concreta. De hecho, el otro día me preguntaba por qué la Comisión de Convivencia solamente estaba centrada en la infancia, ya que tenía que hablar de los temas relacionados con toda la vecindad y con otra serie de cuestiones. Pero claro, nace con la idea de infancia y por eso no son todos los agentes que hay" (V).

Lo que, como se ve, a pesar de la colaboración sostenida, no se había logrado un avance efectivo en un modelo inclusivo de intervención debidamente preventivo en lugar de, como se aprecia, más bien episódico y reactivo. El segundo argumento utilizado remite a que frecuentemente, al crearse redes formales que intervienen de forma local pero que responden a un modelo de planificación y desarrollo de arriba-abajo, con intereses variados y complejos, surgen riesgos de que los objetivos iniciales se desvirtúen, surjan, en lo emocional, desafecciones, en lo relacional pérdidas de confianza y, con todo ello, bloqueos de la comunicación e incluso la ruptura de la colaboración.

De todas formas, y viendo cómo se resolvió este incidente crítico, podemos comprobar la importancia que tienen tanto los objetivos comunes como los vínculos afectivos entre los componentes de una red, también, de carácter formal. Así, en el caso que nos ocupa, los vínculos construidos en la etapa de las redes informales fueron claves para la recuperación de la colaboración dentro de red formal.

"De hecho, entonces nosotros como educadores nos salimos de la Comisión. Lo que pasó es que algunas personas se quejaron... y gente del AMPA y otros vinieron a hablar con nosotros porque creían que teníamos que estar, y les explicamos los motivos..." "Así que pasado un tiempo volvimos a la Comisión" (AS) 
El último hito a destacar corresponde al 2016 y está ligado a la entrada del equipo de la UPV/EHU que está llevando el proyecto al que corresponde este artículo. Nuestra entrada en el escenario del municipio, con el propósito de participar, en colaboración, en el proyecto en cuestión, viene suponiendo una triple oportunidad. Primero, con nuestra incorporación a la Comisión de Convivencia, la de dar visibilidad y reconocimiento a lo que se venía haciendo y estaba poco documentado. Segundo, la de contribuir a relanzar el proceso, hacerlo sostenible e incorporar acciones innovadoras, y más inclusivas, a partir de reflexiones conjuntas en las sesiones de trabajo. Estas últimas, construidas sobre mejoras identificadas en las etapas de colaboraciones informales previas, se concretaron en la incorporación de las voces de la infancia y las familias vulnerables, y el impulso de la participación activa de la ciudadanía en el diseño, el desarrollo y la evaluación de acciones sucesivas. De ese modo, se está haciendo posible la tarea de formalizar y reforzar las relaciones profesionales en el municipio, promoviendo nuevas colaboraciones donde investigación, innovación y formación de futuros profesionales vayan de la mano como motores en la creación y el uso del conocimiento en iniciativas y prácticas inclusivas con la infancia vulnerable y sus familias.

\section{Discusión y conclusiones}

El caso estudiado corrobora que el trabajo colaborativo de todos los agentes con la infancia vulnerable desde una perspectiva inclusiva, es tan urgente como necesario, complejo, difícil de llevar a cabo y, en definitiva, un reto social (Díaz, et al, 2010, Cusso, et al, 2015).

La construcción de una red informal de colaboración comunitaria ha sido, en este caso, fundamental para el desarrollo posterior de una red formal, lo que representa un tema relevante, pero poco tratado en la literatura especializada. Una red informal, propia de una etapa pre-institucional, se caracteriza por la debilidad de elementos relevantes como estructura organizativa, planificación y evaluación a medio y largo plazo que, en teoría, están más definidos en cualquier red formal. Con el tiempo, sin embargo, bajo determinadas condiciones las carencias de lo informal pueden tornarse en fortalezas, mientras que las supuestas fortalezas de lo formalizado también pueden conllevar debilidades. Dadas 
las condiciones y acciones idóneas, el tránsito desde una red informal, todavía operativa, hacia una formal, concretado en la creación de una comisión socioeducativa, por ejemplo, puede conllevar una institucionalización construida y compartida, lo que puede ser crítico.

Concretamente, para generar una referencia importante en un territorio de cara a activar el flujo de información y conocimiento, sacar provecho y fortalecer el capital social, así como generar otro nuevo y compartido. De ese modo, la colaboración en una red socioeducativa, como es nuestro caso, va más allá de intervenciones aisladas y coordinaciones esporádicas y superficiales, haciendo posible las estructuras convenientes y su reconstrucción interna con agentes y acciones socialmente construidas y aplicadas (Díaz y Cívis, 2011), comunidades de prácticas y aprendizaje, si se prefiere. Así pueden crearse espacios habitados por agentes con miradas y actuaciones inclusivas y colectivas, ante el reto de la educación debida a toda la infancia en un determinado territorio (Longás, Civís y Riera, 2008).

Es bien cierto, por lo demás, que las redes socioeducativas no están libres de limitaciones, más si, como es el caso, se persigue un objetivo tan ambicioso como el que se acaba de referir y, sin embargo, el foco de atención es un tanto parcial. Puede ilustrarse con una reflexión a propósito de la red que se articuló, específicamente, en torno al Plan Municipal de Convivencia (2010), como respuesta a la necesidad de intervenir en el territorio con protocolos de intervención a nivel municipal que establecieron los pasos a dar por los diferentes agentes socioeducativos, una vez que se detectaron conductas que alteraban la convivencia. Cabe calificarla, en efecto, como una red específica de resolución de problemas, con la correspondiente planificación e implementación de un proyecto, así como la difusión de información y apropiación comunitaria del mismo (Milward y Provan, 2006). Una temática así construida, incluso asumiendo el propósito de articular diferentes miradas profesionales bajo los auspicios de la inclusión de la infancia vulnerable, puede concentrar la atención sobre el foco de la convivencia, pero, tal vez, no sobre otros asuntos más amplios de una amplia y efectiva inclusión socioeducativa.

De manera que, para conciliar adecuadamente el fondo y la forma, sería necesaria una mayor implicación de los servicios sociales y, así, complementar la red con otros focos, espacios y agentes dentro de ella, ampliando la información, el análisis de necesidades de sujetos y co- 
lectivos de la población, y acometiendo otras acciones coordinadas y novedosas si fuere el caso (Ubieto 2009). Hubiera sido conveniente, en ese sentido, integrar a otros agentes, procurando hacer efectiva una comunidad ampliada de aprendizaje y prácticas compartidas como se dijo. A fin de cuentas, la creación de un espacio que llegue a tener incidencia en las políticas y actuaciones para una inclusión justa y equitativa de la infancia vulnerable (Martínez, 2014), no solo ha de girar sobre problemas de convivencia, aunque también, sino sobre otras necesidades relevantes de la población, igualmente abordables comunitariamente. Algo similar cabe señalar respecto a una efectiva incorporación de la voz de la infancia vulnerable y de sus familias: su participación activa, tanto en los proyectos y su implementación como en la definición de los mismos, es exigible desde los valores inclusivos (Parrilla, Muñoz y Sierra, 2013, Booth y Ainscow, 2015).

Este proyecto, en fase de progreso, está sirviendo para captar mejor la dificultad que comporta la creación de redes socioeducativas. Pone en valor la importancia de fortalecer redes informales y la construcción comunitaria de las mismas, pudiéndose integrar en ellas, además de a los agentes de un territorio, a otros como los universitarios cuyas contribuciones pueden ser convenientes. Concretamente, para acercar y conectar investigación, innovación y respuestas fundadas a determinados problemas sociales. Una contribución relevante a que el capital, que es clave, se aproveche en la construcción y el mantenimiento de redes socioeducativas (Díaz y Cívis, 2011).

El caso concreto estudiado lo corrobora de cara a encarar necesidades sociales de calado, máxime si del referido capital forma parte un determinado modelo de gobernanza local liderado por una clara política municipal en la creación y gestión de entidades sin ánimo de lucro. Tan relevantes son, en la acción socioeducativa con la infancia vulnerable, los servicios y agentes profesionales como lo comunitario y la política. Esa articulación puede llegar a ser lo que marque la diferencia. No solo para afrontar temas específicos y emergentes, sino también tejer una red amplia cuyas políticas y actuaciones integren lo escolar, social, sanitario, cultural, tiempo libre y cualquier asunto que surja del análisis de la realidad, y la voluntad firme de transformarla con una perspectiva comunitaria de inclusión socioeducativa (Marchioni, 2014, Wise, 2016).

Tanto en el ámbito escolar como en el socioeducativo, suele haber distancias acusadas entre la teoría inclusiva (normalización de apoyos 
comunitarios a toda la ciudadanía de una comunidad) y las prácticas llevadas a cabo. Las modalidades de intervención al uso habrían de incluir innovaciones importantes, asentadas en una colaboración en red que refuerce la construcción social de las necesidades, las respuestas a las mismas con proyectos inclusivos y la movilización de acciones comunitarias y sostenidas: seguramente la infancia más vulnerable lo requiere de un modo singular.

El caso estudiado, ilustra bien, asimismo, que problemas tan complejos como los vividos en los noventa y otros posteriores emergentes, pero no menos desafiantes de la infancia vulnerable, han sido comunitariamente afrontados. Como es comprensible, queda camino por hacer movilizando y aplicando esfuerzos colectivos y comunitarios. Una opción de interés puede ser la creación de redes formales con diversos agentes profesionales, servicios y recursos. Es esencial la participación efectiva en ellas de las mismas personas en situación de vulnerabilidad, sujetos y familias, así como que las redes formales creadas se construyan sobre las informales preexistentes. Ello requiere disponer tiempos y espacios idóneos, crear un clima de confianza y participación en la identificación de objetivos comunes, construir modelos horizontales de intervención basados en el respeto y el diálogo y, desde luego, en una cultura con sentido de identidad y responsabilidad social sumando sinergias. Naturalmente, la construcción de tal modelo será un proceso costoso y lento. Exige una reflexión imprescindible sobre y para qué son los proyectos a desarrollar, procurando el equilibrio, de una parte, entre la vertebración teórica y práctica bien fundada y, de otra, hacer frente con actuaciones inmediatas a temas urgentes que lo requieran. En última instancia, los propósitos de una red se refieren tanto a compartir ideas, valores y prácticas con la infancia vulnerable como, simultáneamente, la construcción, desarrollo y despliegue de un determinado modelo comunitario de intervención inclusiva.

\section{Referencias bibliográficas}

Andréu, J. (2000) Las técnicas de análisis de contenido: una revisión actualizada. Fundación Centro Estudios Andaluces, Universidad de Granada, 10 (2) 1-34

Ballester, L. (2014). Redes sociales y escuela. En M.X. March Cerdà \& C. Orte Socias (Coords.). La pedagogía social y la escuela. Los retos socioeducativos de la institución escolar en el siglo XXI (pp.185-206). Barcelona: Octaedro. 
Colaboración y redes socioeducativas para la inclusión de la infancia vulnerable Begoña Martínez Domínguez, Israel Alonso Sáez, Isabel Martínez Domínguez y María José

Booth, T. y Ainscow, M. (2015). Guía para la Educación Inclusiva. Desarrollando el aprendizaje y la participación en los centros escolares. Madrid: FUHEM/OEI.

Brown, J., Collins, A y Duguid, P. (1989) Situated cognition and the culture of learning, Educational Researcher, 18 (1), 32-42.

Castillo, R. (2009). El trabajo en red. Reflexiones desde una experiencia. Zerbitzuan: Gizarte zerbitzuetarako aldizkaria. Revista de servicios sociales, 46, 149-152.

Caride, J. A. (2017). Educación Social, derechos Humanos y sostenibilidad en el desarrollo Comunitario. Teoría de la Educación, 29 (1), 245-272. doi: http://dx.doi. org/10.14201/teoredu2017291245272.

Castel, R. (1997). La metamorfosis de la cuestión social. Una crónica del salariado. Barcelona: Paidós.

Civís, M. \& Longás, J. (2015). La colaboración interinstitucional como respuesta al desafío de la inclusión socioeducativa. Análisis de 4 experiencias de trabajo en red a nivel local en Cataluña. Educación XXI, 18 (1), 213-236. doi: https://doi.org/10.5944/ educXX1.18.1.12318.

Díaz, J., Civís, M., Longás, J. \& López, A. (2010). The Study of Educative Network organization in the City of Barcelona, Spain. The Nou Barris District. International. Journal of Knowledge Society Research. 1(2), 26-37.

Díaz J. y Civís, M. (2011) Redes socioeducativas promotoras de Capital Social en la comunidad: un marco teórico de referencia. Cultura y Educación. Fundación Infancia y Aprendizaje, 23 (3), 415-429.

Escudero, J. M., \& Martínez, B. (2011). Educación inclusiva y cambio escolar. Revista iberoamericana de educación, 55, 85-105

Gutierrez, P., Iglesias., A y Sanchez, C. (2009) HASIERA: un ejemplo de construcción de casos en red. RES. Revista de Educación Social, (24), 1231-1249.

Haurbabesa Lanbide (2011). Con la formación a vueltas: nuevas comprensiones basadas en la investigación cooperativa. RES. Revista de Educación Social, (13), 1-10.

Jolonch, A. (2002). Educació i Infància en risc. Barcelona: Portic.

Kim, K. H. (2014). Community-involved learning to expand possibilities for vulnerable children: A critical communicative, sen's capability, and action research approach. Journal of Mixed Methods Research, 8(3), 308-316. doi: https://doi. org/10.1177/1558689814527877

Longàs, J., Civís, M. y Riera, J. (2008). Asesoramiento y desarrollo de redes socioeducativas locales. Funciones y metodología. Cultura y Educación, 20 (3), 303-324.

Martínez, B. et al. (2015). Percepción de las relaciones entre los profesionales de la educación escolar y social que intervienen con la infancia desprotegida en la comunidad autónoma vasca. Bilbao: UPV/EHU.

Milward, H. B., \& Provan, K. G. (2006). A manager's guide to choosing and using collaborative networks. Washington, DC: IBM Center for the Business of Government.

Marchioni, M. (2014). De las comunidades y de lo comunitario. En Revista Espacios Transnacionales [En línea] No. 3. Julio-Diciembre 2014, Reletran. Disponible en: http://www.espaciostransnacionales.org/tercernumero/reflexiones3/comunidadesycomunitario/ 
Muñoz, M.A., Raposo, M., Doval, I. y Parrilla, A. (2011). Una red local inter-centros para la inclusión educativa. Revista Quaderns Digitals, 69, art. 4.

Organización de Naciones Unidas (ONU). Oficina del Alto Comisionado de las Naciones Unidas para los Derechos Humanos (1989): Convención sobre los Derechos del Niño. Ginebra-Suiza, Publicaciones de Naciones Unidas.

Parrilla, A., Muñoz, M.A. y Sierra, S. (2013). Proyectos educativos con vocación comunitaria. Revista de Investigación en Educación, 11 (3) ,15-31.

Parrilla, Á., y Sierra, S. (2015). Construyendo una investigación inclusiva en torno a las distintas transiciones educativas. Revista Electrónica Interuniversitaria de Formación del Profesorado, 18(1), 161-175.

Prilleltensky, I. (2010). Child wellness and social inclusion: Values for action. American Journal of Community Psychology, 46(1-2), 238-249. doi: https://doi.org/10.1007/ s10464-010-9318-9

Sapon-Shevin, M. (2013). La inclusión real: una perspectiva de justicia social. Revista de Investigación en Educación, 11 (3), 57-70.

SIIS (2017). Estudio diagnóstico de la situación de la infancia y adolescencia en VitoriaGasteiz 2017. Ayuntamiento de Vitoria SIIS. Centro de documentación y estudios 17

Ubieto, J. (2009). El trabajo en red. Barcelona: Gedisa

UNICEF (2107). Situación de la infancia y adolescencia en Euskadi. Hacia un Pacto Vasco por La Infancia y Adolescencia 2017. Bilbao: UNICEF Comité País Vasco

Wenger, E. (2001) Comunidades de práctica, aprendizaje, significado e identidad. Barcelona: Paidós

Wise, S. (2016). Introducing MABL: A New Social Innovations Programme at the University of Melbourne. Children Australia, 41(4), 305-307. doi: https://doi.org/10.1017/ cha.2016.33 